\title{
Autologous Osteochondral Grafts Result in Improved Clinical Outcomes in Patients With Early Knee Osteoarthritis
}

\author{
Yasuaki Nakagawa, M.D., Shogo Mukai, M.D., Kohei Nishitani, M.D., Satoru Murata, M.D., \\ Kentaro Satomi, M.D., Yuki Shinya, M.D., and Ryota Nakamura, M.D.
}

\begin{abstract}
Purpose: To determine whether autologous osteochondral grafts improve clinical outcomes in early knee osteoarthritis (EKOA) and whether the grafts prevent progression of radiographic joint degeneration. Methods: In this study, we identified patients older than 40 years with EKOA having no traumatic events, with grade 0 , 1, or 2 on the KellgrenLawrence $(\mathrm{KL})$ classification and more than grade 3 of cartilage degeneration according to the International Cartilage Repair Society scale. Recipient sites were as follows: 14 patellofemoral (PF3) joint, 18 medial femoral condyle (M1), and 6 lateral femoral condyle (L2). Japanese Orthopedic Association (JOA) score and International Knee Documentation (IKDC) subjective score (SUB) were examined as clinical outcomes. Results: The study included 31 knees in 31 patients who underwent autologous osteochondral grafts for EKOA and more than two years follow-up. The sample included 15 men and 16 women. The mean operative age was 56.0 years, and the mean follow-up period was 76 months (range, 24-170 months). In the preoperative period, JOA and SUB were 73.9 and 49.5, respectively. At follow-up, the JOA and SUB scores were 93.7 and 84.5, respectively. The differences were statistically significant. Progression of KL classification occurred in $0 \%$ of the PF3 group, 17\% of the L2 group, and 33\% of the Ml group. The outcomes of the M1 group significantly became worse $(P=.0381)$ than those of the L2 and the PF3 groups, and the PF3 group significantly maintained good clinical outcomes $(P=.0334)$. Conclusion: Autologous osteochondral grafts for degenerated cartilage improved clinical outcomes of EKOA even if the recipient had cartilage degeneration, and not trauma. The PF3 group maintained significantly good clinical outcomes, and the Ml group became significantly worse.
\end{abstract}

$\mathbf{T}$ here is increasing awareness of the importance of identifying early phases of the degenerative processes in knee osteoarthritis. Defining the phases of the early knee osteoarthritis (EKOA) is important to identify and properly treat patients at risk of progression. ${ }^{1}$

From the Department of Orthopedic Surgery, National Hospital Organization, Kyoto Medical Center (Y.N., S.Mukai, K.S., Y.S., R.N.), Kyoto; the Department of Orthopedic Surgery, Graduate School of Medicine, Kyoto University (K.N.), Kyoto; and Goshohigashi Clinic (S.Murata), Kyoto, Japan.

The authors report that they have no conflicts of interest in the authorship and publication of this article. Full ICMJE author disclosure forms are available for this article online, as supplementary material.

Received January 16, 2021; accepted December 16, 2021

Address correspondence to Yasuaki Nakagawa, M.D., Department of Orthopaedic Surgery, National Hospital Organization Kyoto Medical Center, 1-1 Fukakusa Mukaihata-cho Fushimi-ku, Kyoto 612-8555, Japan. E-mail: yasu0202nakagawa@gmail.com

(C) 2022 THE AUTHORS. Published by Elsevier Inc. on behalf of the Arthroscopy Association of North America. This is an open access article under the CC BY-NC-ND license (http://creativecommons.org/licenses/by-nc-nd/4.0/).

2666-061X/2136

https://doi.org/10.1016/j.asmr.2021.12.016
EKOA may show a diffuse and ill-defined involvement but also originate in the cartilage surrounding a focal lesion. ${ }^{2}$ However, unlike established arthritis, the diagnosis of EKOA is more complicated. Once the disease has manifested, clear signs can be detected through history, clinical symptoms, and radiographic signs. ${ }^{1}$ In the early phase, such characteristic signs and symptoms may still be limited and appear to be sporadic. Moreover, radiographic evaluation cannot detect early changes of the articular cartilage thickness that are required for characterizing established osteoarthritis. Magnetic resonance imaging may detect the whole spectrum of pathological joint tissue changes. Arthroscopy is seldom used as a sole diagnostic tool, but it is often performed in patients suffering from symptomatic focal cartilage defects. ${ }^{2}$ In 2011, Luyten et al. ${ }^{1}$ diagnosed EKOA as the 3 following criteria:

1. At least 2 episodes of knee pain for 10 days in the past year

2. Standard radiographs showing Kellgren-Lawrence (KL) grade 0 to 2 (osteophytes only) 
Table 1. The Various Items in 3 Groups

\begin{tabular}{|c|c|c|c|c|c|c|}
\hline & $\begin{array}{c}\text { Case } \\
\text { Number }\end{array}$ & $\begin{array}{c}\text { Operative } \\
\text { Age }(y)\end{array}$ & $\begin{array}{c}\text { Final Follow-Up } \\
\text { Periods }(\mathrm{mo})\end{array}$ & $\begin{array}{l}\text { Area of Recipient } \\
\text { Site }\left(\mathrm{mm}^{2}\right)\end{array}$ & $\begin{array}{c}\text { Number of } \\
\text { Implanted Plugs }\end{array}$ & $\begin{array}{c}\text { Diameter of } \\
\text { Implanted Plugs }\end{array}$ \\
\hline Medial group & 18 cases & $55.7 \pm 9.3$ & $74.3 \pm 52.3$ & $233.3 \pm 129.6$ & $3.1 \pm 1.4$ & $8.1 \pm 0.9$ \\
\hline Lateral group & 5 cases & $53.3 \pm 9.9$ & $93.7 \pm 55.1$ & $225.0 \pm 133.2$ & $3.4 \pm 1.5$ & $8.4 \pm 0.54$ \\
\hline Patellofemoral group & 8 cases & $53.1 \pm 10.6$ & $60.3 \pm 32.8$ & $217.1 \pm 126.1$ & $2.9 \pm 1.3$ & $8.1 \pm 0.64$ \\
\hline Total & 31 cases & $56.0 \pm 9.3$ & $76.0 \pm 48.7$ & $266.1 \pm 120.9$ & $3.1 \pm 1.4$ & $8.2 \pm 0.71$ \\
\hline
\end{tabular}

Mean value + standard deviation

The 5 items in the 3 groups had no statistically significant differences. Each $P$ value was $>.4448$.

3. International Cartilage Repair Society (ICRS) grades 1 to 4 in at least 2 compartments or grades 2 to 4 in 1 compartment with surrounding softening and swelling in arthroscopy.

More than 20 years have passed since Matsusue et al. ${ }^{4}$ reported autologous osteochondral grafts of the knee in 1993. The use of autologous osteochondral grafts has recently become common for the treatment of small, isolated, well-contained, articular cartilage defects. $^{5-10}$ The technique has been reported to have good clinical outcomes in osteochondral diseases such as osteonecrosis and osteochondritis dissecans. ${ }^{4,10}$ Long-term clinical outcomes after mosaicplasty for a symptomatic osteochondral defect in the knee are good, but they vary greatly depending on age, sex, size, and cause of the lesion. ${ }^{11,12}$

However, few reports have discussed clinical outcomes of autologous osteochondral grafts for EKOA in cartilage degeneration and not in traumatic cartilage injury. The purpose of this study was to determine whether autologous osteochondral grafts improve clinical outcomes in EKOA and whether the grafts prevent progression of radiographic joint degeneration.

\section{Materials and Methods}

Patients who received autologous osteochondral grafts for EKOA from August 2002 to February 2015 were identified. With references to the criteria of Luyten et al. ${ }^{1}$ in 2011 and Madry et al. ${ }^{2}$ in 2016, the patients were diagnosed with EKOA if they had no traumatic events, had a KL grade of 0 to 2, and an ICRS grade of 2 to 4 of cartilage in 1 compartment (focal lesions) in the knee joints in patient 40 years or older. ICRS classification was determined in the surgery. We performed autologous osteochondral grafting for EKOA if conservative treatment (such as nonsteroidal antiinflammatory drugs, hyaluronic acid intraarticular injection, and muscle strength exercises) for more than 3 months had no effect. The inclusion criteria were autologous osteochondral grafts for EKOA, more than 2 years of follow-up, no ligament or patellar instability, and their femorotibial angle was between $170^{\circ}$ and $176^{\circ}$ (normal alignment). The permitted combined operations were partial meniscectomy when the meniscus was ruptured. Patients who underwent ligament surgery or osteotomy to improve knee alignment were excluded from the study. Inflammatory patients were also excluded. All procedures were reviewed and approved by the research ethics committee of our hospital.

\section{Surgical procedure}

Our operative procedures were as follows. First, the knee arthroscopy was performed. In some cases, partial meniscectomy was performed. If the recipient site was the medial femoral condyle, a medial parapatellar approach was used. The curettage of degenerative cartilage in the recipient site were done, and several autologous osteochondral plugs were implanted into the recipient site. For the osteochondral grafts, the Osteochondral Autograft Transfer System (Arthrex, Naples, FL) was mostly used with the open technique. If the recipient site was the lateral femoral condyle, a lateral parapatellar approach was used. If the recipient site was the trochlea or patella, a medial parapatellar approach was used, and we implanted plugs obliquely to create the curvature of the articular surface of both the patella and trochlea.

The postoperative rehabilitation program was as follows: Range of motion exercise started 3 days after mosaicplasty; partial weightbearing gait started in week 2 in patellofemoral joints and week 3 or 4 in femorotibial joints, and full weightbearing gait started in week 5 or 7 . The patient donor site was either the lateral or medial side of the femoral trochlea (relatively non-weightbearing surface). In general, the donor defects were filled with bone chips from the recipient sites to subchondral bone level, not to the articular cartilage surface.

Table 2A. JOA Scores in 3 Groups

\begin{tabular}{llcc}
\hline & $\begin{array}{c}\text { Number } \\
\text { of Cases }\end{array}$ & $\begin{array}{c}\text { Preoperative } \\
\text { Period (Points) }\end{array}$ & $\begin{array}{c}\text { Follow-Up } \\
\text { Period (Points) }\end{array}$ \\
\hline Medial group & 18 cases & $70.0 \pm 11.3$ & $93.9 \pm 10.2$ \\
Lateral group & 5 cases & $73.3 \pm 11.3$ & $93.3 \pm 10.2$ \\
$\begin{array}{l}\text { Patellofemoral } \\
\quad \text { group }\end{array}$ & 8 cases & $75.4 \pm 12.1$ & $93.9 \pm 7.1$ \\
Total & 31 cases & $73.9 \pm 11.7$ & $93.7 \pm 8.9$ \\
\hline
\end{tabular}


Table 2B. IKDC Subjective Scores in 3 Groups

\begin{tabular}{llcc}
\hline & $\begin{array}{c}\text { Number } \\
\text { of Cases }\end{array}$ & $\begin{array}{c}\text { Preoperative } \\
\text { Period (Points) }\end{array}$ & $\begin{array}{c}\text { Follow-Up } \\
\text { Period (Points) }\end{array}$ \\
\hline Medial group & 18 cases & $46.4 \pm 11.3$ & $83.8 \pm 15.8$ \\
Lateral group & 5 cases & $46.9 \pm 18.6$ & $87.2 \pm 11.1$ \\
$\begin{array}{c}\text { Patellofemoral } \\
\text { group }\end{array}$ & 8 cases & $49.8 \pm 15.6$ & $86.6 \pm 6.6$ \\
\begin{tabular}{l} 
Total \\
\hline
\end{tabular} & 31 cases & $49.5 \pm 13.6$ & $84.5 \pm 13.1$ \\
\hline
\end{tabular}

IKDC, International Knee Documentation Committee.

Mean value \pm standard deviation. Significant differences were observed in IKDC subjective scores between the preoperative period and follow-up period in all three groups, including total scores. Preoperative and follow-up IKDC subjective scores in 3 each groups had no significant differences.

The operative age, follow-up period, area of the recipient site, the number of implanted plugs, and the diameter of implanted plugs for the 3 groups were recorded in Table 1. The symptoms of the patients' knees were evaluated according to the following criteria: the knee scoring system of the Japanese Orthopedic Association (JOA) score ${ }^{13}$ and the IKDC subjective score and objective score. ${ }^{14}$ The JOA score evaluates 4 items: ability to walk ( 30 points), ability to climb up and down stairs (25 points), range of motion ( 35 points), and joint swelling (10 points). Each knee joint can achieve a maximum score of 100 points on the JOA scale. We also examined changes in the KL classification of their knee radiographs from their surgeries to their final follow-up.

To compare the clinical outcomes, we divided the recipient sites into 3 groups: medial, lateral, and patellofemoral. To compare the changes of KL classification, we divided the recipient sites into 3 groups: Ml group, L2 group, and PF3 group.

The Mann-Whitney U-test (JOA and IKDC subjective scores) and the $\chi^{2}$ test (IKDC objective score and the worseness of KL classification) were used to perform statistical analyses of the various scores. The level of statistical significance was set to $P<.05$.

\section{Results}

A total of 31 cases and 31 knees were included from August 2002 to February 2015. Almost surgeries were performed by 1 senior orthopaedic surgeon (Y.N.). The follow-up rate was $81 \%$. We examined 11 right and 20 left knees of 15 men and 16 women. The mean operative age was 56.0 years (range, $40-71$ years), and the mean follow-up period was 76 months (range, 24-170 months). The permitted combined operations were 4 cases of arthroscopic partial medial meniscectomy, and 1 case of arthroscopic partial lateral meniscectomy. According to the recipient site, 31 patients were assigned to 3 of the following groups: 18 cases in the medial group ( 13 in medial femoral condyle [MFC] only, 4 in MFC and trochlea, and 1 in MFC and lateral tibia), 5 cases in the lateral group ( 2 in lateral femoral condyle (LFC) only, 2 in LFC and trochlea, and 1 in lateral tibia), and 8 cases in the patellofemoral group ( 5 in trochlea only, and 3 in trochlea and patella).

We also examined changes in the KL classification. Because we could examine each compartment of the same knee separately, we categorized the patients into 3 groups different than the clinical assessment: 18 knees in the Ml group (13 in MFC only, 4 in MFC and trochlea, and 1 in MFC and lateral tibia), 6 knees in the L2 group ( 2 in lateral femoral condyle [LFC] only, 2 in LFC and trochlea, 1 in lateral tibia and 1 in MFC and lateral tibia), and 14 knees in the $\mathrm{PF} 3$ group (5 in trochlea only, 3 in trochlea and patella, 4 in MFC and trochlea and 2 in LFC and trochlea).

The operative age, follow-up period, area of the recipient site, the number of the implanted plugs, and the diameter of the implanted plugs for the 3 groups were recorded in Table 1 . The mean number of implanted plugs was 3.1 (range, 1-6), and the mean diameter of implanted plugs was $8.2 \mathrm{~mm}$ (range, 6-10 $\mathrm{mm})$. The 5 items that were described in Table 1 in the 3 groups had no statistically significant differences.

With respect to the clinical outcomes, JOA scores during the preoperative and follow-up periods in the 3 groups are shown in Table $2 \mathrm{~A}$, and IKDC subjective scores at the preoperative and follow-up periods in the 3 groups are shown in Table 2B. Significant differences were observed in JOA and IKDC subjective scores between the preoperative period and follow-up period in all 3 groups, including total scores. Preoperative JOA scores in each of the 3 groups had no significant differences, and the follow-up JOA scores also did not have any significant differences. Similarly, IKDC subjective scores had no significant differences.

Table 3 shows IKDC objective scores during the preoperative and follow-up periods in total cases. IKDC objective scores at follow-up were significantly better than that during the preoperative period. We found 8 cases with less than abnormal findings at follow-up; they were as follows: 5 in MFC only, 1 in LFC only, 1 in trochlea only, and 1 in trochlea and LFC. Three cases in MFC only get worse than preoperative period. However, only l case needed reoperation (total knee

Table 3. IKDC Objective Scores in Preoperative and FollowUp Periods in Total Cases

\begin{tabular}{lcc}
\hline & Preoperative Period & Follow-Up Period \\
\hline Normal & 0 cases & 5 cases \\
Nearly normal & 3 cases & 18 cases \\
Abnormal & 25 cases & 6 cases \\
Severely abnormal & 3 cases & 2 cases \\
\hline
\end{tabular}

IKDC, International Knee Documentation Committee.

IKDC objective scores at follow-up were significantly better than that during the preoperative period. 
Table 4. The comparison with the worseness of KL classification in 3 groups

\begin{tabular}{lccc}
$\begin{array}{l}\text { Change of KL } \\
\text { Classification } \\
\text { (Preoperative }\end{array}$ & $\begin{array}{l}\text { M1 Group } \\
\text { (18 Knees) }\end{array}$ & $\begin{array}{l}\text { L2 Group } \\
\text { (6 Knees) }\end{array}$ & $\begin{array}{l}\text { PF3 Group } \\
\text { (14 Knees) }\end{array}$ \\
\hline fo Follow-Up) & 5 knees & 1 knee & 5 knees \\
2 to 2 & 7 & 4 & 9 \\
1 to 2 & 1 & 0 & 0 \\
1 to 3 & 1 & 0 & 0 \\
2 to 3 & 2 & 1 & 0 \\
2 to 4 & 2 & 0 & 0 \\
Worse cases & $6(33 \%)$ & $1(16.7 \%)$ & $0(0 \%)$ \\
\hline
\end{tabular}

KL, Kellgren-Lawrence; M1, medial compartment; L2, lateral compartment; PF3, patellofemoral compartment.

arthroplasty), and she belonged to the Ml group; this detail description was written in the case presentation.

Table 4 shows the comparison of aggravation of KL classification between preoperative and follow-up periods in the three groups. Using $\chi^{2}$ test, the $\mathrm{Ml}$ group was significantly worse than the other 2 groups $(P=$ $.0381)$, and the PF3 group was significantly maintained, compared with the other 2 groups $(P=.0334)$; this answers the second question of whether the grafts prevent the progression of radiographic joint degeneration.
The following were 2 case examples.

\section{Patient 1}

This was a 52-year-old male who underwent mosaicplasty for the treatment of EKOA on his left knee. He belonged to the PF3 group. The recipient area was $450 \mathrm{~mm}^{2}$ in his trochlea (Fig $1 \mathrm{~A}$ ), and he had 4 osteochondral plugs with a diameter of $8 \mathrm{~mm}$ implanted into the recipient sites (Fig 1B). Three donor plugs were in the lateral trochlea, and 1 donor plug was in the medial trochlea (Fig 1B). The preoperative lateral and skyline radiographies of his left knee showed osteophytes in his medial and lateral PF joints (Figs IC and 1D). At 114 months after his mosaicplasty, the lateral and skyline radiographies of the left knee were the same (Figs $1 \mathrm{E}$ and $\mathrm{IF}$ ). The KL grade was 2 during the preoperative period and at follow-up. IKDC subjective score was 63.6 points during the preoperative period and 79.2 points at 114 months after mosaicplasty.

\section{Patient 2}

This was a 64-year-old female who underwent mosaicplasty for the treatment of EKOA on her right knee. The recipient area was $150 \mathrm{~mm}^{2}$ (Fig 2A) in her MFC, and 2 osteochondral plugs with a diameter of 8 $\mathrm{mm}$ were implanted into the recipient sites (Fig 2B).
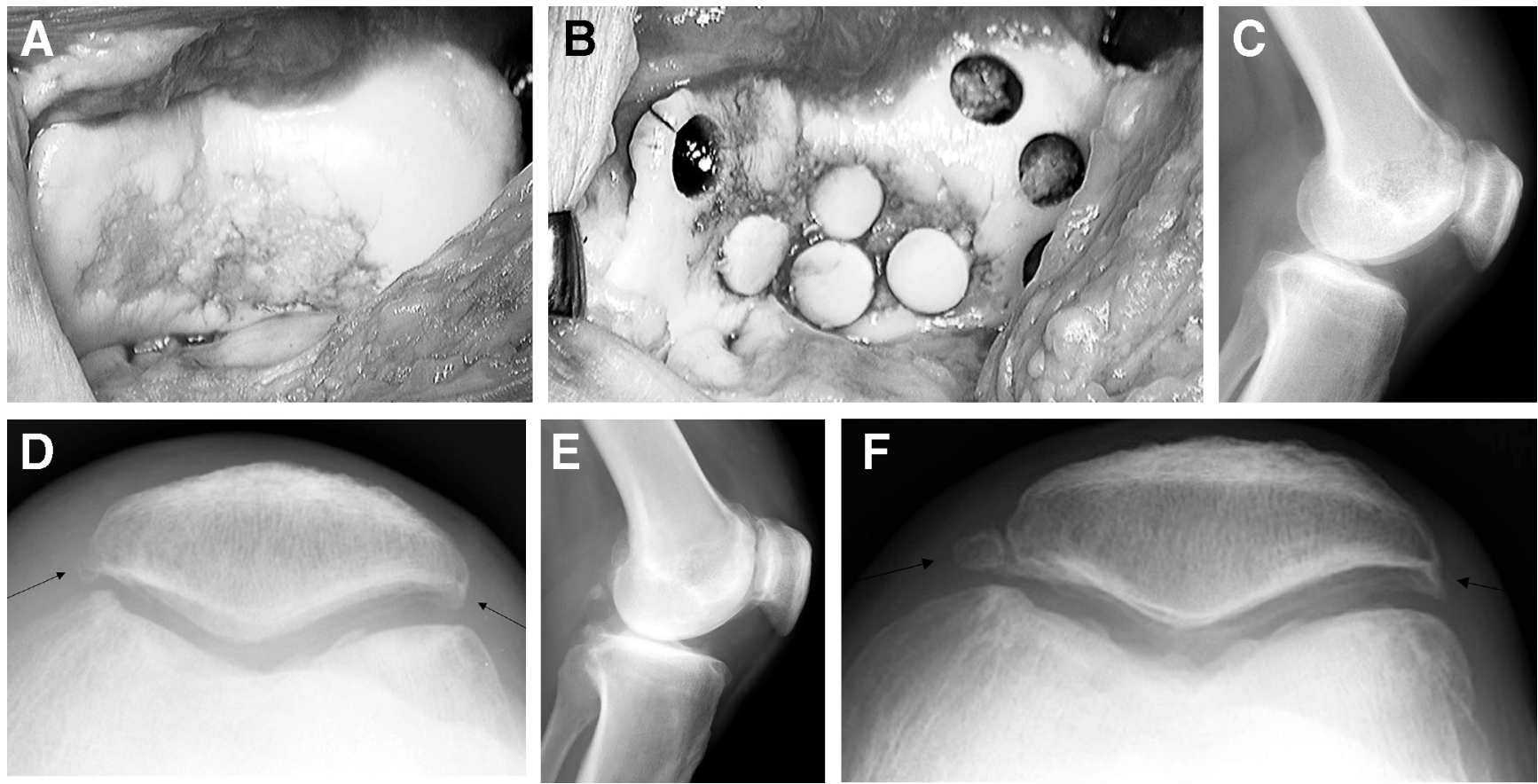

Fig 1. Patient 1 belonged to the patellofemoral group. (A) A 52-year-old male who underwent mosaicplasty early knee osteoarthritis for his left knee. The recipient area was $450 \mathrm{~mm}^{2}$ in his trochlea. (B) Four osteochondral plugs with a diameter of 8 mm were implanted into the recipient sites. (C) The preoperative lateral radiography. (D) The preoperative skyline radiography of the left knee showed osteophytes (arrows) in his medial and lateral patellofemoral joints. (E) At 114 months after his mosaicplasty, the lateral radiography (F) and skyline radiography of the left knee was the same (arrows). His preoperative Kellgren-Lawrence grade was 2, and that at follow-up was 2. His International Knee Documentation Committee subjective score was 63.6 points before surgery, and at 114 months after mosaicplasty it was 79.2. 

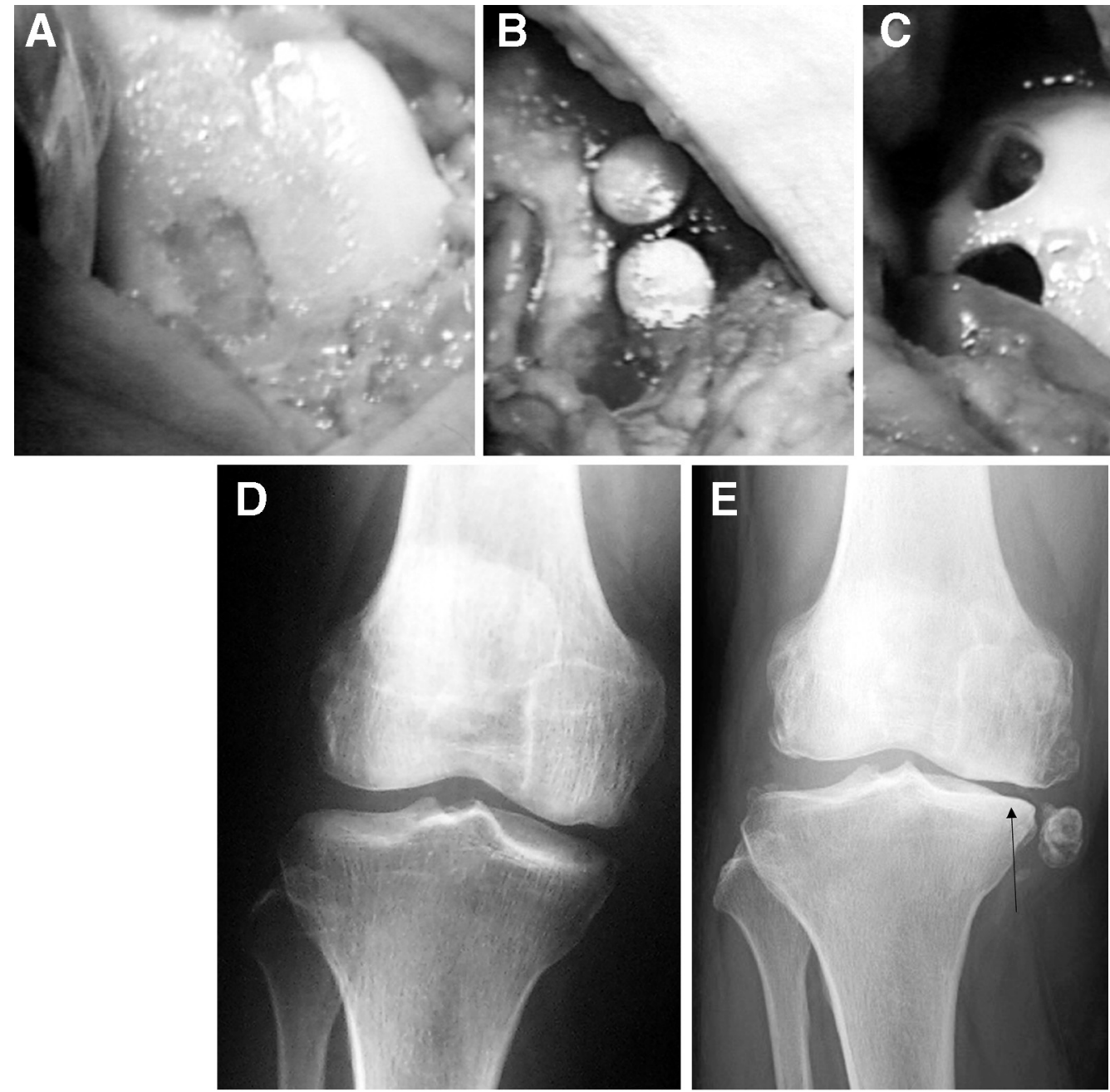

Fig 2. Patient 2 belonged to the medial group. (A) A 64-year-old female who underwent mosaicplasty for early knee osteoarthritis on her right knee. The recipient area was $150 \mathrm{~mm}^{2}$ in her medial femoral condyle (MFC). (B) Two osteochondral plugs with a diameter of $8 \mathrm{~mm}$ were implanted into the recipient sites. (C) Two donor plugs in lateral trochlea were taken. (D) The preoperative anteroposterior radiography of the right knee showed osteophytes in her MFC. (E) At 14l months after her mosaicplasty, the anteroposterior radiography of the right knee showed narrowing of her medial compartment (arrow). Her preoperative Kellgren-Lawrence grade was 2, and that at follow-up was 3. Her International Knee Documentation Committee subjective score was 57.1 points before surgery and 141 months after mosaicplasty, it was 33.1.

Two donor plugs in the lateral trochlea were taken (Fig 2C). The preoperative radiography of the right knee showed osteophytes in the MFC (Figs 2D and 2E); at 141 months after her mosaicplasty, the radiograph of the right knee showed narrowing of her medial compartment (Figs 2F and 2G). The preoperative KL grade was 2, and it was 3 at follow-up. The patient's preoperative IKDC subjective score was 57.1 points, and it was 33.1 points at 141 months after mosaicplasty. At that time, she had right total knee arthroplasty (TKA) because of severe knee pain. In this study, only 1 case converted to TKA.

\section{Discussion}

In this study we observed that the autologous osteochondral grafts did improve clinical outcomes, even if the recipient had cartilage degeneration with no trauma, and second, the Ml group significantly became worse than the other 2 groups, and the PF3 group significantly maintained good clinical outcomes, compared with the other 2 groups.

Madry et al. ${ }^{2}$ defined EKOA as being present if 3 of the following criteria were fulfilled: pain in the knee, standard radiograph with $\mathrm{KL}$ grade of 0 to 2 , and at least 1 of the following 2 structural criteria: arthroscopic findings of cartilage lesions, magnetic resonance imaging findings demonstrating articular cartilage degeneration, meniscal degeneration, or subchondral bone marrow lesions. Therefore EKOA was diagnosed if the patients had no traumatic events, were older than 40 years, having a KL grade of 0 to 2, and an ICRS grade of 2 to 4 of cartilage in 1 compartment in the knee joints in 
this study. In the natural course of early osteoarthritis, Roemer et al. ${ }^{15}$ emphasized that effusion synovitis, a reflection of inflammatory activity including joint effusion and synovitis thickening, might play a role in the development of cartilage lesions in knees without $\mathrm{OA}$ in the future. Cartilage defects, bone marrow edema, and meniscal tears correlated with persistent symptoms, at least in patients at high risk of developing OA. ${ }^{16}$ Moreover, bone marrow edema and meniscal lesions were associated with increased incidence of cartilage defects and loss of cartilage. ${ }^{16}$ In 1979, Higano et al. $^{17}$ reported that advanced osteoarthritis had a significantly higher odds ratio compared to nonosteoarthritis and early osteoarthritis with the tibial plateau angle and medial tibial cortical bone thickness. The current study showed the clinical outcomes of only autologous osteochondral grafts for EKOA.

With respect to the long-term outcomes of mosaicplasty, Solheim et al. ${ }^{18}$ reported that long-term (minimum 15 years) mosaicplasty resulted in better and clinically relevant outcomes than microfracture in articular cartilage defects $\left(2-5 \mathrm{~cm}^{2}\right)$ of the distal femur of the knee in patients aged 18 to 50 years. However, few reports available on mosaicplasty outcomes for EKOA. Brittberg et al. ${ }^{19}$ stressed the need to identify trigger mechanisms that start generalized loss of cartilage matrix and induce subchondral bone changes and concomitant synovial pathology, to maximize treatment methods for biological repair in degenerative aging joints. The current study showed that autologous osteochondral grafts improved clinical outcomes of EKOA, even in recipient cartilage degeneration, not trauma.

In a comparison of outcome among different recipient sites for mosaicplasty, the PF joint was worse than medial or lateral femorotibial joint. ${ }^{7,11}$ The reason may be the difficulty in accurately reproducing the curvature of the articular surface of both the patella and trochlea. ${ }^{20}$ However, we implanted plugs obliquely to create the curvature of the articular surface of both the patella and trochlea (Fig 1B). Culvenor et al. ${ }^{21}$ reported that the PF compartment seemed to be at particular risk for early OA after anterior cruciate ligament reconstruction. However, in the current study, the Ml group significantly became worse than the other 2 groups, and the PF3 group significantly maintained good clinical outcomes, compared with the other 2 groups. It may relate to recipient cartilage degeneration in the current study, but not trauma. Pelletier et al. ${ }^{22}$ reported that meniscal damage and bone changes were the features most closely associated with the greatest subregional cartilage volume loss, and for the first time, joint space narrowing was strongly associated with cartilage loss in the central areas of plateaus and condyles. That also may explain the significant maintenance in the PF joint compared with the medial or lateral femorotibial joint.
There have been several reports of outcomes of autologous chondrocyte implantation (ACI) for EKOA. In an animal model, Shinhan et al reported that a matrix-associated ACI group of sheep achieved significantly better results in both macroscopic and histological examination. ${ }^{23}$ In human studies, at 5 years after treatment with ACI for EKOA, 92\% of patients were functioning well and were able to delay the need for joint replacement. ${ }^{24}$ At more than 10 years' follow-up ACI, there were 3 total knee replacements $(4.3 \%))^{25}$ and at the 15-year evaluation there were 17 failures $(21.2 \%)$ and 6 total knee replacements $(7.5 \%)$ in the ACI group. ${ }^{26}$ The failure rates of ACI treatment up to 9 years varied from $8 \%$ to $27.3 \%$, and the rate of conversion to TKA rate was $2.5 \%$ to $6.5 \% .{ }^{27}$ In the current series, the worse cases of clinical outcomes were 10 cases $(32.3 \%)$ in the JOA score, 8 cases $(25.8 \%)$ in the IKDC objective score, and 7 cases $(22.6 \%)$ in the KL classification. Only 1 case $(3.2 \%)$ showed a conversion to TKA. There were no major differences between the current series and other ACI reports. ${ }^{24,25}$

\section{Limitations}

The small sample size (31 cases and 31 knees, including 5 cases with arthroscopic partial meniscectomy), the shorter mid-term follow-up (76 months: range, 24 to 170 months), not large follow-up ratio $(81 \%)$ and an observational study (not a randomized clinical trial) were the most important limitations of the current study. And 3 subgroup sample size were also small. Because this is a level 4 study, with no control group, we cannot insist that autologous osteochondral grafts directly led to improve clinical outcomes or prevent progression of osteoarthritis.

\section{Conclusion}

Autologous osteochondral grafts for degenerated cartilage improved clinical outcomes of EKOA even if the recipient had cartilage degeneration and not trauma. The PF3 group maintained significantly good clinical outcomes, and the Ml group became significantly worse.

\section{References}

1. Luyten FP, Denti M, Filardo G, Kon E, Engebretsen L. Definition and classification of early osteoarthritis of the knee. Knee Surg Sports Traumatol Arthrosc 2011;20: 401-406.

2. Madry H, Kon E, Condello V, et al. Early osteoarthritis of the knee. Knee Surg Sports Traumatol Arthrosc 2016;24: 1753-1762.

3. Kellgren JH, Lawrence JS. Radiological assessment of osteo-arthrosis. Ann Rheum Dis 1957;16:494-502.

4. Matsusue Y, Yamamuro T, Hama H. Arthroscopic multiple osteochondral transplantation to the chondral defect in the knee associated with anterior cruciate ligament disruption: a case report. Arthroscopy 1993;9:318-321. 
5. Bobic V. Arthroscopic osteochondral autograft transplantation in anterior cruciate ligament reconstruction: A preliminary clinical study. Knee Surg Sports Traumatol Arthrosc 1996;3:262-264.

6. Hangody L, Kish G, Karpati Z, Udvarhelyi I, Szigeti I, Bely M. Mosaicplasty for the treatment of articular cartilage defects: Application in clinical practice. Orthopedics 1998;21:751-756.

7. Jakob RP, Franz T, Gautier E, Mainil-Varlet P. Autologous osteochondral grafting in the knee: Indication, results, and reflections. Clin Orthop 2002;401:170-184.

8. Nakagawa Y, Matsusue Y, Ikeda N, Asada Y, Nakamura T. Osteochondral grafting and arthroplasty for end-stage osteochondritis dissecans of the capitellum. A case report and review of the literature. Am J Sport Med 2001;29:650-655.

9. Yamashita F, Sakakida K, Sufu F, Takai S. The transplantation of an autogenic osteochondral fragment of osteochondral dissecans of the knee. Clin Orthop 1985;201:43-50.

10. Nakagawa Y, Matsusue Y, Nakamura T. Osteochondral graft transplantation for steroid-induced osteonecrosis of the femoral condyle. Lancet 2003;362:402.

11. Hangody L, Dobos J, Balo E, Panics G, Hangody LR, Berkes I. Clinical experiences with autologous osteochondral mosaicplasty in an athletic population: A $17-$ year prospective multicenter study. Am J Sport Med 2010;38:1125-1133.

12. Solheim E, Hegna J, Oyen J, Harlem T, Strand T. Results at 10 to 14 years after osteochondral autografting (mosaicplasty) in articular cartilage defects in the knee. Knee 2013;20:287-290.

13. Nakagawa Y, Mukai S, Yamada S, et al. Short-term effects of highly-bioavailable curcumin for treating knee osteoarthritis: A randomized, double-blind, placebo-controlled prospective study. J Orthop Sci 2014;19:933-939.

14. Brittberg M, Aglietti A, Gambardella R, et al. ICRS cartilage injury evaluation package. http://www.cartilage. org/. Accessed August 2000.

15. Roemer FW, Guermazi A, Felson DT, et al. Presence of MRI-detected joint effusion and synovitis increases the risk of cartilage loss in knees without osteoarthritis at 30month follow-up: The MOST study. Ann Rheum Dis $2011 ; 70: 1804-1809$.

16. Favero M, Ramonda R, Goldring MB, Goldring SR, Punzi L. Early knee osteoarthritis. RMD Open 2015;1(Suppl 1): e000062.

17. Higano Y, Hayami T, Omori G, Koga Y, Endo K, Endo N. The varus alignment and morphologic alterations of proximal tibia affect the onset of medial knee osteoarthritis in rural Japanese women case control study from the longitudinal evaluation of Matsudai knee osteoarthritis survey. J Orthop Sci 2016;21:166-171.

18. Solheim E, Heqna J, Strand T, Harlem T, Inderhaug E. Randomized study of long-term (15-17 years) outcome after microfracture versus mosaicplasty in knee articular cartilage defects. Am J Sports Med 2018;46: 826-831.

19. Brittberg M, Gomoll AH, Canseco JA, Far J, Lind M, Hui J. Cartilage repair in the degenerative ageing knee. A narrative and analysis. Acta Orthopaedica 2016;87:26-38 (e Suppl 363).

20. Arendt E, Berruto M, Filardo G, et al. Early knee osteoarthritis of the patellofemoral joint. Knee Surg Sports Traumatol Arthrosc 2016;24:1836-1844.

21. Culvenor AG, Collins NJ, Guermazi A, et al. Early knee osteoarthritis is evident one year following anterior cruciate ligament reconstruction. A magnetic resonance imaging evaluation. Arthritis Rheum 2015;67:946-955.

22. Pelletier JP, Raynauld JP, Berthiaume MJ, et al. Risk factors associated with the loss of cartilage volume on weight-bearing areas in knee osteoarthritis patients assessed by quantitative magnetic resonance imaging: A longitudinal study. Arthritis Res Ther 2007;9:R74.

23. Schinhan M, Gruber M, Dorotka R, Pilz M, Stelzeneder D, Chiari C, Rossler N, Windhager R, Nehrer S. Matrixassociated autologous chondrocyte transplantation in a compartmentalized early stage of osteoarthritis. Osteoarthritis Cart 2013;21:217-1370.

24. Minas T, Gomoll AH, Solhpour S, Rosenberger R, Probst C, Bryant T. Autologous chondrocyte implantation for joint preservation in patients with early osteoarthritis. Clin Orthop Relat Res 2010;468:147-157.

25. Ebert JR, Fallon M, Ackland TR, Janes GC, Wood DJ. Minimum 10-year clinical and radiological outcomes of a randomized controlled trial evaluating 2 different approaches to full weightbearing after matrix-induced autologous chondrocyte implantation. Am J Sports Med 2020;48:133-142.

26. Knutsen G, Drogset JO, Engebretsen L, et al. A randomized multicenter trial comparing autologous chondrocyte implantation with microfracture: Long-term follow-up at 14 to 15 years. J Bone Joint Surg Am 2016;98:1332-1339.

27. Windt TS, Vonk LA, Brittberg M, Saris DBF. Treatment and prevention od (early) osteoarthritis using articular cartilage repair-Fact or fiction? A systematic review. Cartilage 2013;4:5S-12S (Suppl 3). 